\title{
Inertial and 3D-odometry fusion in rough terrain - Towards real 3D navigation
}

\author{
P. Lamon, R. Siegwart \\ Swiss Federal Institute of technology (EPFL) \\ Lausanne, Switzerland \\ pierre.lamon@epfl.ch,roland.siegwart@epfl.ch
}

\begin{abstract}
Many algorithms related to localization need good pose prediction in order to produce accurate results. This is especially the case for data association algorithms, where false feature matches can lead to the localization system failure. In rough terrain, the field of view can vary significantly between two feature extraction steps, so a good position prediction is necessary to robustly track features. This paper presents a method for combining dead reckoning sensor information in order to provide an initial estimate of the six degrees of freedom of a rough terrain rover. An inertial navigation system (INS) and the wheel encoders are used as sensory inputs. The sensor fusion scheme is based on an extended information filter (EIF) and is extensible to any kind and number of sensors. In order to test the system, the rover has been driven on different kind of obstacles while computing both pure 3D-odometric and fused INS/3Dodometry trajectories. The results show that the use of the INS significantly improves the pose prediction.
\end{abstract}

Keywords - rough terrain, INS, odometry, information filter

\section{INTRODUCTION}

A good pose estimate is essential for an autonomous mobile robot because position is used by most of the navigation tasks and algorithms running onboard. The first step of localization consists in the integration of high frequency dead reckoning sensors to predict vehicle location. The second phase uses some form of absolute sensing mechanism for extracting relevant features in the environment and reset the position prediction. One of the big challenges of this update is to find correspondences between the current and previously extracted landmarks and features. This task needs good pose prediction in order to provide reliable results and limit false matches. This requirement is even more important when the robot travels on a cluttered terrain, where the field of view can vary significantly between two features extraction steps.

The Inertial Navigation Systems (INS ${ }^{1}$ ) provide direct measurements of the dynamic of the system and are selfcontained. For these reasons they are used in many applications for predicting the robot's position and orientation during the first localization step. The INS were first used in aerospace applications and a large part of the literature refers to them. The availability of low-cost solidstate sensors allowed using INS for ground applications

\footnotetext{
${ }^{1}$ An INS is generally composed of a triad of accelerometers (velocity
} rate sensors) and gyroscopes (angular rate sensors) such as road vehicle and mobile robots. Nevertheless, these sensors provide less accurate position information and their implementation on ground vehicles is more difficult. Indeed, trajectories are less smooth on the ground where the system has to deal with chocks and more vibrations.

Many research works are related to road vehicles applications where INS are used to provide higher update rate of the position between two consecutive GPS data acquisition. Furthermore, they can also be used for estimating the wheel diameter changes and the vehicle sideslip [1,2]. Barshan and Durrant-Whyte [3] showed that low-cost INS can improve the system performance and can be applied to mobile robotics if an accurate sensor model is provided. A method for combining data from gyroscopes and odometry is presented in [4]. Scheding and al. [5] present interesting results for an underground mining vehicle. They show clearly how inertial sensors can be used to correct for non-systematic errors due to soil irregularities when fused with other sensors such as wheel encoders and laser scanners.

However, most of the published works show results in two dimensions and deal with the estimation of the planar position and orientation only. Furthermore, the target environment is generally flat and the structure of the soil can be known beforehand. This allows developing relatively accurate vehicle models, which lead to good odometric information.

The intent of this paper is to propose a method for combing 3D-odometric and inertial information in order to provide a robust three-dimensional initial estimate of the six degrees of freedom of a rough terrain rover. This method, based on an extended information filter, is presented in section three. Section two shortly describes the rover used for the experiments and the applied 3Dodometry method. Section four presents the experimental results, validating the theory. Finally, sections five and six will present ongoing research and conclude the paper.

\section{BACKGROUND}

\section{A. The rough terrain rover}

The Autonomous System Lab (at EPFL) developed a six-wheeled off-road rover called Shrimp, which shows excellent climbing capabilities thanks its passive mechanical structure. The most recent prototype is depicted in Fig. 1 and a detailed description can be found in [6]. 


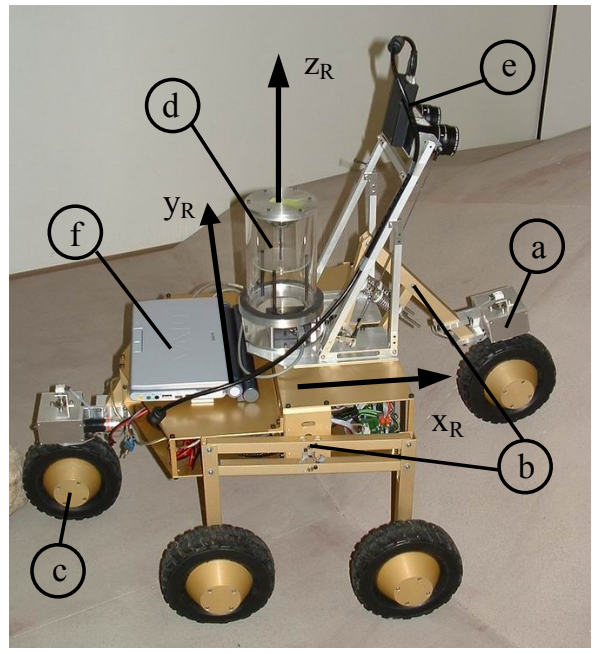

Figure 1. A picture of the latest prototype, called Solero. The inertial navigation system is hidden in the rover's body and therefore is not visible. (a) steering servo mechanism, the same is used for the rear wheel (b) passively articulated bogie and spring suspended front fork (all equipped with absolute angular sensors) (c) one of the 6 motorized wheels (with encoders, speed, torque and position control capabilities) (d) omnidirectional vision system (e) stereo-vision module, orientable around the tilt axis (f) main computation unit

This rover can intrinsically adapt to a large range of obstacles and therefore can move smoothly across rough terrain. This behavior has many advantages when dealing with onboard sensors. In particular, it allows limited wheel slip and vibration. The quality of the odometric information and the ratio signal/noise for the inertial sensors are significantly improved in comparison with rigid structures such as four-wheel drive rovers. Thus, both odometry and INS integration techniques can be accounted for position estimation.

\section{B. 3D-odometry}

Although odometry is widely used indoors (2D), its application is limited in rough terrain (3D) because the wheels are more likely to slip and the position error can grow quickly. The 3D-odometry extends the standard (2D) odometry to the three-dimensional space and is described thoroughtfully in [7]. This technique computes the position increments $(d x, d y, d z)$ and the orientation $(d \psi)$ of the robot from the bogie wheel encoders, an inclinometer and the bogies angular sensors. Because it accounts for ground slope discontinuities, this method produces much better estimates than the standard method on uneven terrain. However, the 3D-odometry still assumes no-slip and is therefore subject to performance loss in very cluttered terrains. The experimental results show that the INS allows correcting accidental and non-systematic errors, which significantly improves the position estimate.

\section{PROBABILISTIC SENSOR FUSION SCHEME}

For this application an extended information filter (EIF) is used for combining the information coming from the $3 \mathrm{D}$ odometry and the inertial unit. This formulation of the Kalman filter has interesting features. Its mathematical expression is well suited for implementing a distributed sensor fusion scheme and allows to easily extending the system to any kind and number of sensors [8]. Fig. 2 depicts the schematics of the fusion process.

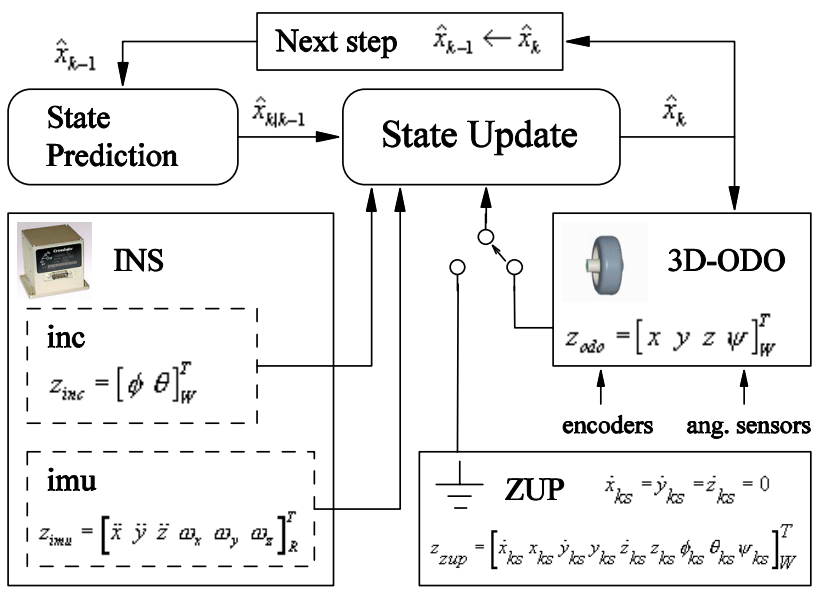

Figure 2. The EIF sensor fusion scheme, easily extendable to more sensors. The INS is divided into two logical sensors: an inclinometer and an inertial measurement unit. When the robot is stopped, at time $k_{\mathrm{s}}$, the ZUP (Zero Update Position) becomes active. This allows guarantying fast convergence of the INS biases and no drift while the robot is stopped

The information state vector and the information matrix are updated according to:

$$
\begin{aligned}
& \hat{y}(k \mid k)=\hat{y}(k \mid k-1)+\sum_{j \in S} H_{j}(k)^{T} R_{j}^{-1}(k) z_{j}^{\prime}(k) \\
& P^{-1}(k \mid k)=P^{-1}(k \mid k-1)+\sum_{j \in S} H_{j}(k)^{T} R_{j}^{-1}(k) H_{j}(k)
\end{aligned}
$$

where the set $\mathrm{S}=\{\mathrm{imu}$, inc, odo/zup $\}$ and

$$
\begin{aligned}
& z^{\prime}(k)=z(k) \quad \text { for the linear filter, otherwise } \\
& z_{j}^{\prime}(k)=z_{j}(k)-\left(h_{j}[k, \hat{x}(k \mid k-1)]-\nabla_{x} h_{j}[k, \hat{x}(k \mid k-1)] \hat{x}(k \mid k-1)\right)
\end{aligned}
$$

The inverse covariance and the information state vector are predicted as:

$$
\begin{aligned}
& P(k \mid k-1)=F(k) P(k-1 \mid k-1) F(k)^{T}+Q(k) \\
& \hat{y}(k \mid k-1)=P^{-1}(k \mid k-1) F(k) P(k-1 \mid k-1) \hat{y}(k-1 \mid k-1)
\end{aligned}
$$

Finally the state vector may be obtained from:

$$
\hat{x}(k \mid k)=P(k \mid k) \hat{y}(k \mid k)
$$

\section{A. The sensor models}

For each sensor the matrices $\mathrm{H}_{\mathrm{j}}$ and $\mathrm{R}_{\mathrm{j}}$ (with $\mathrm{j} \in \mathrm{S}$ ) must be defined. The measurement models $\mathrm{H}_{\mathrm{j}}$ will be presented in this section while the content of the covariance matrices $\mathrm{R}_{\mathrm{j}}$ will be discussed in the experimental results section.

1) The inertial unit model: The position, velocity and attitude can be computed by integrating the readings from the INS. However, both accelerometers and gyros can be influenced by bias errors. Even if these errors are small they will cause an unbounded growth in the error of integrated measurements. The velocity and the attitude error will diverge proportionally over time and the position to the square of time. The accelerometers measurements are thus modeled as: 


$$
\left[\begin{array}{c}
\ddot{x} \\
\ddot{y} \\
\ddot{z}
\end{array}\right]_{R}^{z}=\Gamma_{W R}\left[\begin{array}{c}
\ddot{x} \\
\ddot{y} \\
\ddot{z}
\end{array}\right]_{W}+\left[\begin{array}{l}
b_{a x} \\
b_{a y} \\
b_{a z}
\end{array}\right]_{R}+v_{a}
$$

and the gyros as:

$$
\begin{aligned}
& {\left[\begin{array}{l}
\omega_{x} \\
\omega_{y}
\end{array}\right]^{z}=\left[\begin{array}{l}
\omega_{x} \\
\omega_{y}
\end{array}\right]+\left[\begin{array}{l}
b_{\omega x} \\
b_{\omega y}
\end{array}\right]+v_{\omega}} \\
& \omega_{z}^{z}=\left(1+\Delta_{\omega z}\right)\left[\omega_{z}+b_{\omega z}\right]+v_{\omega z}
\end{aligned}
$$

$\Gamma_{W R}$ is the rotation matrix that transforms values expressed in the world-fixed coordinates system $W$ into the robot's coordinates system $R$ (see Fig. 1). This matrix is a function of the angles $\phi$ (roll), $\theta$ (pitch) and $\psi$ (yaw). The $b$ 's and $v$ 's are the biases and the white measurements noises respectively. Because the variations of measurement of the z-gyro can be large, the scaling error $\Delta_{\omega z}$ has been added to the model.

The equations (5) and (7) are non-linear and the first order Taylor expansion is used to provide:

$$
\begin{aligned}
& {\left[\begin{array}{c}
\ddot{x} \\
\ddot{y} \\
\ddot{z}
\end{array}\right]_{R}^{z}=\bar{\Gamma}_{W R}\left[\begin{array}{c}
\ddot{x} \\
\ddot{y} \\
\ddot{z}
\end{array}\right]_{W}+\nabla\left[\bar{\Gamma}_{W R} \cdot \bar{a}\right]\left[\begin{array}{l}
\phi \\
\theta \\
\psi
\end{array}\right]+\left[\begin{array}{c}
b_{a x} \\
b_{a y} \\
b_{a z}
\end{array}\right]_{R}+v_{a}} \\
& \text { with } \quad \bar{a}=\left[\begin{array}{lll}
\overline{\ddot{x}} & \overline{\ddot{y}} & \bar{z}-g
\end{array}\right]^{T} \\
& \omega_{z}^{z}=\left(1+\bar{\Delta}_{\omega z}\right) \omega_{z}+\left(1+\bar{\Delta}_{\omega z}\right) b_{\omega z}+\left(\bar{\omega}_{z}+\bar{b}_{\omega z}\right) \Delta_{\omega z}+v_{\omega z}
\end{aligned}
$$

where the bars denotes operating point values and $g$ is the gravitational constant, which has to be removed before integrating the accelerations. The $\mathrm{H}_{\mathrm{imu}}$ matrix can be constructed using (6), (8), (9). The $\mathrm{H}_{\text {inc }}$ matrix is the identity matrix because the inclinometer directly measures $\phi$ and $\theta$.

Because the INS is not placed exactly at the center of the robot, it is subject to centripetal accelerations due to the angular rates. They have to be subtracted from the measurements in order to consider the accelerations related to the center of the robot, which is used as the reference point by all the other sensors. The centripetal contribution $\boldsymbol{c}_{i}$ for each accelerometer is:

$$
\begin{aligned}
& \mathbf{c}_{i}=\boldsymbol{\omega} \times\left(\boldsymbol{\omega} \times \mathbf{r}_{i}\right)+\dot{\boldsymbol{\omega}} \times \mathbf{r}_{i} \\
& \text { with } \boldsymbol{\omega}=\left[\omega_{x} \omega_{y} \omega_{z}\right]^{T} \text { and } i \in\{x, y, z\}
\end{aligned}
$$

where $\boldsymbol{r}_{\mathrm{i}}$ is the position of each accelerometer $i$ with respect to the center of the robot.

2) The odometry measurement model: The robot used for this research is a skid-steered rover and the natural and controlled motion is in the forward direction. Thus, the errors due to wheel slip and wheel diameters variations have much more effect in the $\mathrm{x}-\mathrm{z}$ plane than along the $\mathrm{y}$ direction. Therefore, scaling errors $\left(\Delta_{\mathrm{ox}}\right.$ and $\left.\Delta_{\mathrm{oz}}\right)$ have been introduced only for the $\mathrm{x}$ and $\mathrm{z}$-axes:

$$
\begin{aligned}
& x^{z}=\left(1+\Delta_{o x}\right) x+v_{o x} \quad y^{z}=y+v_{y} \quad z^{z}=\left(1+\Delta_{o z}\right) z+v_{o z} \\
& \psi^{z}=\psi+v_{\psi}
\end{aligned}
$$

where the $v$ 's are the white measurements noises. The $\mathrm{H}_{\text {odo }}$ matrix is obtained using the first order Taylor decomposition of (11).

\section{B. The state prediction model}

The state prediction model is of the form:

$$
\begin{aligned}
& {\left[\begin{array}{l}
\mathbf{x}_{\mathbf{x}} \\
\mathbf{x}_{\mathbf{y}} \\
\mathbf{x}_{\mathbf{z}} \\
\mathbf{x}_{\mathbf{b a}} \\
\mathbf{x}_{\mathbf{\omega}} \\
\mathbf{x}_{\mathbf{b} \boldsymbol{\omega}} \\
\mathbf{x}_{\Delta}
\end{array}\right]_{k+1}=\left[\begin{array}{ccccccc}
F_{x} & & & & & & \\
& F_{y} & & \ldots & & 0 & \\
& & F_{z} & & & & \\
& \vdots & & F_{b a} & & \vdots & \\
& & & & F_{\omega} & & \\
& 0 & & \ldots & & F_{b \omega} & \\
& & & & & & F_{\Delta}
\end{array}\right]\left[\begin{array}{l}
\mathbf{x}_{\mathbf{x}} \\
\mathbf{x}_{\mathbf{y}} \\
\mathbf{x}_{\mathbf{z}} \\
\mathbf{x}_{\mathbf{b a}} \\
\mathbf{x}_{\omega} \\
\mathbf{x}_{\mathbf{b} \boldsymbol{\omega}} \\
\mathbf{x}_{\Delta}
\end{array}\right]_{k}+\left[\begin{array}{l}
w_{\mathbf{x}} \\
w_{\mathbf{y}} \\
w_{\mathbf{z}} \\
w_{\mathbf{b a}} \\
w_{\omega} \\
w_{\mathbf{b} \boldsymbol{\omega}} \\
w_{\Delta}
\end{array}\right]_{k}}
\end{aligned}
$$

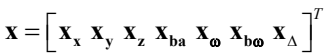

$$
\begin{aligned}
& =\left[\ddot{x} \dot{x} x, \ddot{y} \dot{y} y, \ddot{z} \dot{z} z, b_{a x} b_{a y} b_{a z}, \omega_{x} \phi \omega_{y} \theta \omega_{z} \psi,\right. \\
& \left.b_{\omega x} b_{\omega y} b_{\omega z}, \Delta_{\omega z} \Delta_{o x} \Delta_{o z}\right]^{T}
\end{aligned}
$$

The accelerations, angular rates, biases and scaling errors are changing randomly depending on the motion commands, time and other non-modeled parameters. However, they cannot be considered as pure white noise because they are highly time correlated. Instead they can be modeled as first order Gauss-Markov processes whose auto-correlation function is:

$$
R_{f}(t)=\sigma^{2} e^{-\tau|t|}
$$

where $1 / \tau$ is the correlation time and $\sigma^{2}$ the variance of the process. Such a process can also be considered as a low pass filter, with $\tau$ being the time constant. The discrete functions of the first and second integral of such a process can be easily computed using the inverse Laplace operator. They are developed in the following equation:

$$
\left[\begin{array}{l}
x_{1} \\
x_{2} \\
x_{3}
\end{array}\right]_{k+1}=\left[\begin{array}{ccc}
e^{-\tau h} & 0 & 0 \\
\left(1-e^{-\tau h}\right) / \tau & 1 & 0 \\
\left(\tau h-1+e^{-\tau h}\right) / \tau^{2} & h & 1
\end{array}\right]\left[\begin{array}{l}
x_{1} \\
x_{2} \\
x_{3}
\end{array}\right]_{k}=\Phi(\tau, h) \cdot\left[\begin{array}{l}
x_{1} \\
x_{2} \\
x_{3}
\end{array}\right]_{k}
$$

where $x_{2}$ and $x_{3}$ are respectively the first and second integral of the Gauss-Markov process $x_{l}$ and $h$ is the sampling time. The covariance matrix $Q$ is then derived by computing the expectations $E\left\{x_{i} x_{j}\right\}$ with $\mathrm{i}, \mathrm{j}=1 . .3$.

Thus, because the accelerations, biases and scaling errors are modeled as Gauss-Markov processes, one can write:

$$
\begin{aligned}
& F_{x}=\Phi\left(\tau_{x}, h\right) \quad F_{y}=\Phi\left(\tau_{y}, h\right) \quad F_{z}=\Phi\left(\tau_{z}, h\right) \\
& Q_{x}=E\left\{w_{\mathbf{x}} w_{\mathbf{x}}^{T}\right\} \quad Q_{y}=E\left\{w_{\mathbf{y}} w_{\mathbf{y}}^{T}\right\} \quad Q_{z}=E\left\{w_{\mathbf{z}} w_{\mathbf{z}}^{T}\right\} \\
& F_{b a}=\operatorname{diag}\left(e^{-\tau_{b a x}}, e^{-\tau_{b a y}}, e^{-\tau_{b a z}}\right) \quad Q_{b a}=E\left\{w_{\mathbf{b a}} w_{\mathbf{b a}}^{T}\right\} \\
& F_{b \omega}=\operatorname{diag}\left(e^{-\tau_{b o x}}, e^{-\tau_{b o y}}, e^{-\tau_{b o z}}\right) \quad Q_{b \omega}=E\left\{w_{\mathbf{b} \omega} w_{\mathbf{b}_{\omega}}^{T}\right\} \\
& F_{\Delta}=\operatorname{diag}\left(e^{-\tau_{\Delta \Delta z}}, e^{-\tau_{\Delta \Delta x}}, e^{-\tau_{\Delta o z}}\right) \quad Q_{\Delta}=E\left\{w_{\Delta} w_{\Delta}^{T}\right\}
\end{aligned}
$$

where $\operatorname{diag}(a, b, c)$ refers to a diagonal matrix composed of the elements $\mathrm{a}, \mathrm{b}$ and $\mathrm{c}$.

The derivation of $F_{\omega}$ is more tedious because the dynamic of $\mathbf{x}_{\omega}$ is non-linear. Furthermore, the small-angle approximation cannot be made because the robot is subject 
to move on rough terrain, where the angular variations can be of high amplitude ${ }^{2}$. Equation (16) describes the nonlinear state transition of $\mathbf{x}_{\omega}$ :

$$
\begin{aligned}
& \omega_{x_{k+1}}=\omega_{x_{k}} e^{-\tau_{\omega_{x}} h}, \quad \omega_{y_{k+1}}=\omega_{y_{k}} e^{-\tau_{\omega_{y}} h}, \quad \omega_{z_{k+1}}=\omega_{z_{k}} e^{-\tau_{\omega_{z}} h} \\
& \phi_{k+1}=f_{1}\left(\mathbf{x}_{\omega}\right)=\phi_{k}+h\left(\left(\omega_{y} \sin (\phi)+\omega_{z} \cos (\phi)\right) \tan (\theta)+\omega_{x}\right) \\
& \theta_{k+1}=f_{2}\left(\mathbf{x}_{\omega}\right)=\theta_{k}+h\left(\omega_{y} \cos (\phi)-\omega_{z} \sin (\phi)\right) \\
& \psi_{k+1}=f_{3}\left(\mathbf{x}_{\omega}\right)=\psi_{k}+h\left(\omega_{y} \sin (\phi)+\omega_{z} \cos (\phi)\right) / \cos (\theta)
\end{aligned}
$$

The linearized $6 \times 6$ matrix $F_{\omega}$ can then be obtained by computing the Jacobians of the $f$ 's functions and including the terms for the angular rates.

Despite the large dimension of the state vector, the state prediction computation can be done online with a nonoptimized code (less than $1 \mathrm{~ms}$ on a $666 \mathrm{MHz}$ processor).

\section{EXPERIMENTAL RESULTS}

The rover depicted in section II is equipped with a VG400-CC inertial measurement unit from Crossbow. It is a six-axis measurement system designed to measure linear accelerations and rotation rates along/around three orthogonal axes. Furthermore, it includes a digital signal processor running a Kalman filter for directly estimating the roll and pitch angles in both static and dynamic conditions. For the sensor fusion scheme this unit is divided into two logical sensors (see Fig. 2). One is considered as an inclinometer and the other provides angular rates and accelerations measurements.

\section{A. $\quad$ Setting the $Q$ matrix}

The values in $Q$ are difficult to set because they are based on the knowledge of the system and the operating conditions. It is not possible to measure these values because they can vary significantly during locomotion in rough terrain. However, some simple rules can be applied for guessing how the parameters are related to each other. Furthermore, taking some margin on the variances estimations will allow accounting for a larger range of situations. The INS provides direct measurements of the dynamic of the system and updates the state vector frequently. Thus, the datasheet of the sensor can be used for setting some of the state transition model parameters. They are presented in Table I together with some comments on how they have been selected.

TABLE I. PARAMETERS FOR THE STATE TRANSITION MODEL

\begin{tabular}{|l|l|}
\hline $\begin{array}{l}\tau_{\ddot{x}}=0.6, \tau_{\ddot{y}}=0.1 \\
\tau_{\ddot{z}}=0.1\end{array}$ & $\begin{array}{l}\text { The experiments show that the y and } \\
\mathrm{z} \text {-axes are more subject to vibration. } \\
\text { Thus they have to be more filtered. }\end{array}$ \\
\hline$\sigma_{\ddot{x}}^{2}=\sigma_{\ddot{z}}^{2}=0.005$ & $\begin{array}{l}\text { There is a direct relation between the } \\
\text { rover's control commands and the } \mathrm{x} \\
\text { and } \mathrm{z} \text { accelerations, their variance is } \\
\text { then set larger than for y. }\end{array}$ \\
$\sigma_{\ddot{y}}^{2}=0.003$ & $\begin{array}{l}\text { The biases are changing slower than } \\
\text { the accelerations over time. Thus, } \\
\text { their time constants are set smaller. }\end{array}$ \\
\hline$\tau_{b a x}=\tau_{b a y}=0.016$ & \\
$\tau_{b a z}=0.002$ &
\end{tabular}

${ }^{2}$ The propagation of the Euler angles with time is fully developed in [9].

\begin{tabular}{|l|l|}
\hline $\begin{array}{l}\sigma_{b a x}^{2}=\sigma_{b a y}^{2}=0.2 \\
\sigma_{b a z}^{2}=0.11\end{array}$ & $\begin{array}{l}\text { The experiments showed that these } \\
\text { values should be set larger than the } \\
\text { one prescribed in the INS datasheet. }\end{array}$ \\
\hline$\tau_{\omega x}=\tau_{\omega y}=1$ & $\begin{array}{l}\omega_{\mathrm{z}} \text { is governed by the command } \\
\text { signals to the rover, it is then subject } \\
\text { to change more rapidly than } \omega_{\mathrm{x}, \mathrm{y}}\end{array}$ \\
$\tau_{\omega z}=3$ & $\begin{array}{l}\text { These values are set to the square of } \\
\text { the half of the maximum biases for } \\
\text { the gyros }(2 \sigma), \text { which can be found } \\
\text { in the INS datasheet. }\end{array}$ \\
\hline$\sigma_{b \omega x}^{2}=\sigma_{b \omega y}^{2}=0.0006$ \\
$\sigma_{b \omega z}^{2}=0.012$ & $\begin{array}{l}\text { Same remarks as for the acceleration } \\
\text { biases. }\end{array}$ \\
\hline$\tau_{\Delta w z}=3 \cdot 10^{-4}$ & \\
$\sigma_{\Delta w z}^{2}=3 \cdot 10^{-5}$ & $\begin{array}{l}\text { They have been determined } \\
\text { experimentally; the final results are } \\
\text { not very sensitive to them. }\end{array}$ \\
\hline$\tau_{\Delta o x}=\tau_{\Delta o z}=2$ &
\end{tabular}

\section{B. Setting the $R_{j}$ matrices}

In order to set the variances for the INS sensor, the rover has been driven along a straight trajectory at different velocities and on different types of soil while collecting statistics. The experiments showed that the variances don't change significantly upon velocity and terrain type. This is thanks the passive mechanical structure of Solero. Finally, the worst-case set of variances has been selected for $\mathrm{R}_{\mathrm{imu}}$ and $\mathrm{R}_{\text {inc }}$.

The sensor model for the odometry is much more tedious to assess because the robot is subject to drive across all kind of terrain and soil such as sand, rock and grass. It is very difficult to classify all types of terrain and configurations and to associate the corresponding variances. Instead, we set the uncertainty of the odometric information being proportional to the acceleration undergone by the rover. Indeed, slip mostly occurs in rough terrain during obstacle overcoming, while the robot is subject to accelerations. Furthermore, at constant speed, the acceleration is zero and thus does not bring much information. In this case, the position estimation can rely only on odometry. For the same reasons, the variance for the yaw angle has been set proportional to the angular rate. Thus, the $\mathrm{R}_{\text {odo }}$ matrix can be written as:

$$
R_{\text {odo }}=\bar{\Gamma}_{R W} C_{R} \bar{\Gamma}_{R W}^{T}
$$

where $\Gamma_{R W}$ is the transformation matrix converting quantities from the robot frame into the world frame and the matrix $\mathrm{C}_{\mathrm{R}}$ :

$$
C_{R}=\left[\begin{array}{cccc}
k_{x}\left(1+\ddot{x}_{R}^{z}-g_{x}\right) & & & 0 \\
\vdots & k_{y}\left(1+\ddot{y}_{R}^{z}-g_{y}\right) & & \vdots \\
0 & \ldots & k_{z}\left(1+\ddot{z}_{R}^{z}-g_{z}\right) & \\
k_{\psi}\left(1+\omega_{z}^{z}\right)
\end{array}\right]
$$

$k_{\mathrm{x}}, k_{\mathrm{y}}, k_{\mathrm{z}}$ and $k_{\psi}$ are constants set empirically and $g_{\mathrm{x}}, g_{\mathrm{y}}$ and $g_{z}$ are the gravitational components in the rover-fixed frame. $k_{\mathrm{x}}$ and $k_{\mathrm{z}}$ have been set larger than $k_{\mathrm{y}}$ because Solero is a skid-steered rover: the motion commands affect the $\mathrm{x}-\mathrm{z}$ position, $y$ is not directly controllable. This set of constants has been tested and validated during the experiments performed on different types of terrains. 


\section{Experimental validation}

In order to test the sensor fusion method, the robot has been driven forward across different experimental setups during a fixed interval of time. Then the pure 3D-odometry and filtered trajectories have been compared. By filtered trajectory we mean the trajectory build out of the position estimates computed by the EIF filter. We have repeated the same experiment several times and measured the final position of the robot for each run

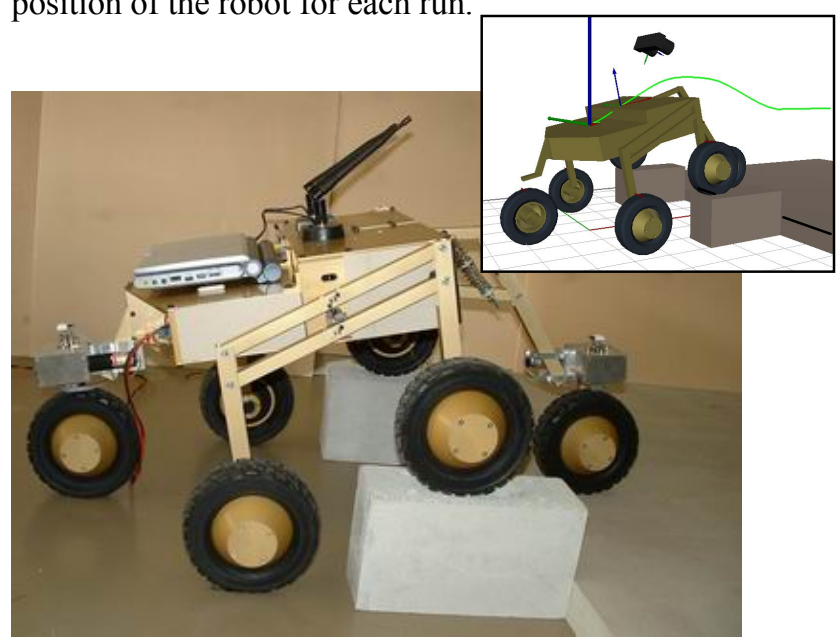

Figure 3. Picture of one of the experimental setups along with the corresponding 3D model used for analysing the results. Because the dimensions of the obstacles are known, we can measure precisely the true maximal and final position heights. In this case the maximal height is $135 \mathrm{~mm}$ and the final height is $45 \mathrm{~mm}$. That kind of obstacle is very difficult to negociate for a wheeled rover because of the sharp edges and the low friction coefficient.

Fig. 3 depicts the most difficult obstacle configuration the rover has been faced to during the experiments. Because of a low friction coefficient between the wheels and the obstacle, a lot of slip occurs during the step climbing. Furthermore, the robot literally bounces on the ground when the rear bogie wheels go down from the obstacle. The raw $\mathrm{x}$ and $\mathrm{z}$-accelerometer signals are plotted in Fig. 4. The shocks occurring during the experiment are easily identified when looking at the z-accelerometer plot.

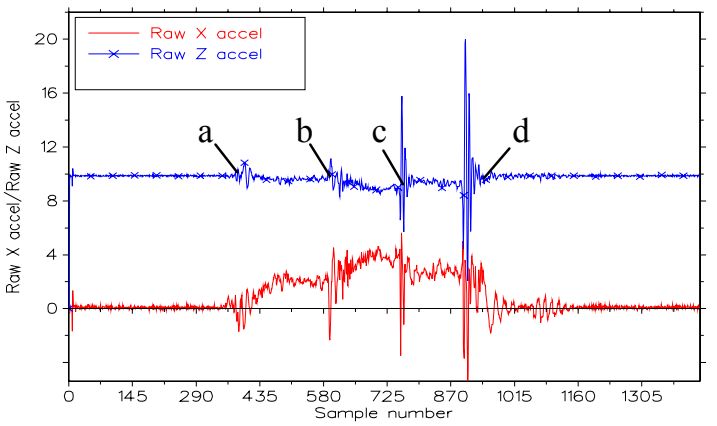

Figure 4. The raw $x$ and $z$-accelerometer signals. The amplitude of the accelerations can reach values higher than $1 \mathrm{~g}$ (a) front bogie wheels climbing on the obstacle (b) rear bogie wheels climbing (c) front bogie wheels going down the obstacle (d) rear bogie wheels going down.

Table II reports the final measurements together with the relative position errors. The third run, highlighted in the table, will be used as example for the next two figures.
TABLE II. EXPERIMENTAL MEASUREMENTS

\begin{tabular}{|c|c|c|c|c|c|c|c|c|}
\hline \multicolumn{9}{|c|}{ Experimental values (mm) } \\
\hline \multicolumn{3}{|c|}{ Measured } & \multicolumn{3}{|c|}{ 3D-odometry only } & \multicolumn{3}{|c|}{ Filtered } \\
\hline $\mathrm{x}$ & $\mathrm{y}$ & Z & $\mathrm{x}$ & $\mathrm{y}$ & Z & $\mathrm{x}$ & $\mathrm{y}$ & Z \\
\hline 1020 & 4 & 45 & 1150 & 88 & 40 & 1160 & 17 & 44 \\
\hline 1025 & 7 & 45 & 1149 & 66 & 40 & 1152 & 38 & 40 \\
\hline 1030 & 5 & 45 & 1182 & 58 & 38 & 1184 & 18 & 44 \\
\hline 1030 & 2 & 45 & 1149 & 31 & 33 & 1150 & 29 & 34 \\
\hline 1025 & 1 & 45 & 1152 & 35 & 36 & 1152 & 16 & 37 \\
\hline Aver: & & & $13 \%$ & $1771 \%$ & $17 \%$ & $13 \%$ & $776 \%$ & $11 \%$ \\
\hline
\end{tabular}

The relative error along the $\mathrm{x}$-axis is the same for both the $3 \mathrm{D}$-odometry and the filtered trajectories. This result can be explained: it is because wheel slip mainly occurs when the robot starts climbing the obstacle at constant speed, while the trajectory is smooth. During this phase, the accelerometers don't detect velocity change and therefore can't help correcting the position. On the other hand, when the rover goes down the obstacle, the $\mathrm{z}$ accelerometer information allows correcting the trajectory and the relative error along the z-axis is only $11 \%$ instead $17 \%$. Fig. 5 depicts this correction nicely. For all the experiments the filtered final $\mathrm{z}$-coordinate is always closer to the true height of $45 \mathrm{~mm}$.

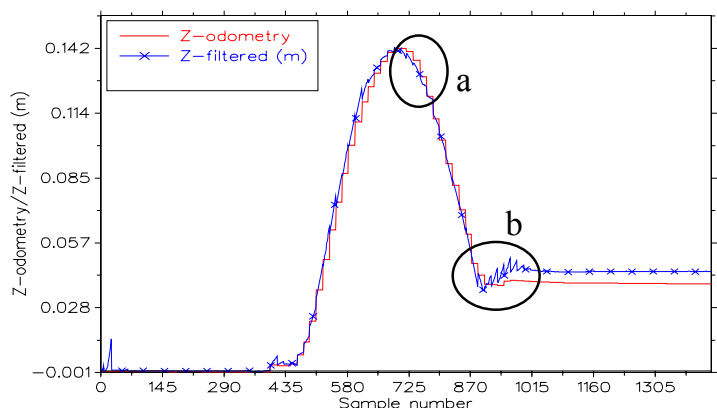

Figure 5. The $\mathrm{z}$ trajectories for the third run (see Table II). The ellipses (a) and (b) show the correction occuring when the front, respectively rear, bogie wheels go down from the step.

The error in the y-direction is mostly due to the heading (yaw) error occurring during asymmetric wheels slip. The odometry is very sensitive to this effect and the yaw estimation can vary significantly even for small slip. Fig. 6 shows how the yaw gyro helps correcting the heading. The result is a fair diminution of the relative error along the $y-$ axis (see Table II).

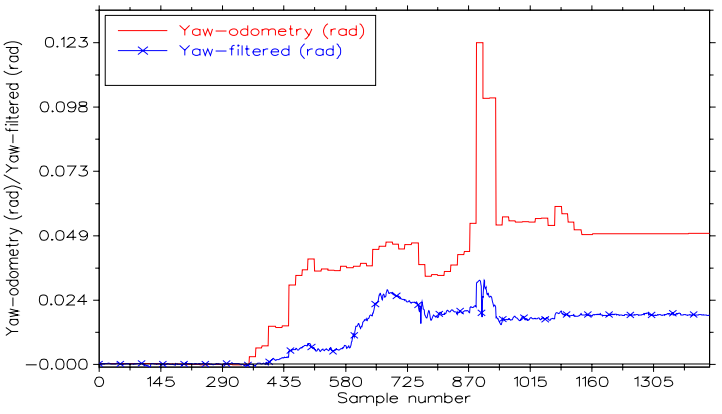

Figure 6. The yaw angles estimates for the third run (the true final angle is close to zero). The yaw gyroscope (measuring the angular rate around $\mathrm{z}$ ) allows correcting for asymmetric slip. 
The errors along the $\mathrm{x}$-axis being the same, it is interesting to consider the absolute errors in the y-z plane. Fig. 7 shows that the final positions computed with the sensor fusion algorithm are systematically closer to the true position than the pure 3D-odometry estimations.

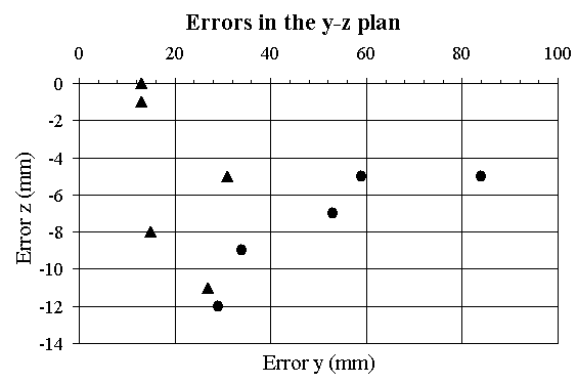

Figure 7. Errors in the $y-z$ plane. The triangles represent the filtered values and the circles, the pure 3D-odometry estimations.

For testing the system in a more general case, the rover has been driven twenty times across the scene depicted in Fig. 8. Each time, the operator remote controlled the rover in order to close the loop. For each run, the final error of the filtered trajectory was smaller than the pure-odometry.

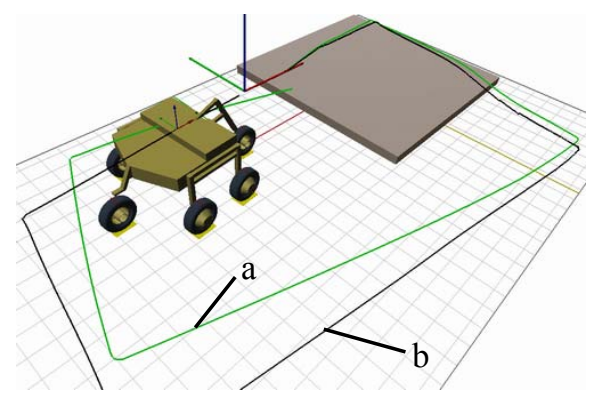

Figure 8. Comparison between pure odometry (a) and filtered trajectory (b). The final error $\left[\varepsilon_{\mathrm{x}}, \varepsilon_{\mathrm{y}}, \varepsilon_{\mathrm{z}}, \varepsilon_{\psi}\right]$ (in meters and degrees) is respectively $\left[0.16,0.142,0.014,18^{\circ}\right]$ and $\left[0.06,0.029,0.012,1.2^{\circ}\right]$ for this run.

\section{FUTURE WORK}

In order to reduce errors due to wheel slip, a quasi-static model of Solero together with a torque-optimization method that minimize slip have been developed [10]. The simulations show promising results and the system is ready to be implemented on the rover for real testing. This type of control is better than the speed control currently implemented on the rover because it is based on the physical model of the rover. It should not only minimize odometric error but also reduce the overall energy consumption and increase the robot's climbing performance.

After the prediction phase, the localization phase consists in extracting specific features in the environment and corrects the position prediction. For this purpose, the visual motion estimation method presented in [11] will be used together with the interest point-matching algorithm developed in $[12,13]$. When fed with a good initial pose estimate, the visual motion estimation algorithm produces better matches and results, especially in rough terrain. This new source of motion information will be easily integrated into the system thanks the versatile sensor fusion scheme.

\section{CONCLUSION}

In this paper, a method for combining dead reckoning sensor information in order to provide an initial estimate of the six degrees of freedom of an off-road rover has been presented. The experimental results showed that the inertial navigation system helped to correct odometric errors and significantly improved the pose estimate; especially when the robot is overcoming sharp-shaped obstacles.

A good initial pose estimate helps the localization algorithms to provide accurate position information. Thus, this work should allow expanding the range of speed and surface roughness over which the rover should be able to go and keep track of its position. It will certainly help to go towards real 3D navigation for outdoor robots.

\section{ACKNOWLEDGMENT}

The author would like to thank Mr. Burnier, Michaud and Terrien for their help with the robot's hardware, Lacroix, Jung and Mallet for the visual motion estimation implementation. This project has been funded by the EPFL's research council and the ESA Solero project.

\section{REFERENCES}

[1] Bevly, D.M., Sheridan, R., Gerdes, J.C., "Integrating INS sensors with GPS velocity measurements for continuous estimation of vehicle sideslip and tire cornering stiffness", Proceedings of the American Control Conference, Volume 1, June 2001.

[2] Wada, M., Kang Sup Yoon, Hashimoto, H., "High accuracy multisensor road vehicle state estimation", 26th Annual Conference of the IEEE Industrial Electronics Society, IECON 2000,Oct. 2000.

[3] B. Barshan, H. F. Durrant-Whyte, "Inertial Navigation Systems for Mobile Robots", IEEE Transac. on Robotics and Automation, 1995.

[4] J. Borenstein, L. Feng, "Gyrodometry: A new method for combining data from gyros and odometry in mobile robots", In the proceedings of the 1996 IEEE International Conference on Robotics and Automation, Minneapolis, April 1996.

[5] Scheding, S., Dissanayake, G., Nebot, E.M., Durrant-Whyte, H., "An experiment in autonomous navigation of an underground mining vehicle", IEEE Transactions on Robotics and Automation, Volume: 15 Issue: 1 , Feb. 1999

[6] Siegwart R., Lamon P., Estier T., Lauria M., Piguet R., "Innovative design for wheeled locomotion in rough terrain", Journal of Robotics and Autonomous Systems, Elsevier, vol 40/2-3 p151-162.

[7] Lamon P., Siegwart R., "3D-Odometry for rough terrain - Towards real 3D navigation", Proceedings of IEEE International Conference on Robotics and Automation (ICRA 2003), Taipei, May 2003.

[8] J. Manyika and H. Durrant-Whyte, "Data fusion and sensor management: A decentralized information-theoretic approach", Ellis Horwood Limited, 1994.

[9] D. H. Titterton, J. L. Weston, "Strapdown inertial navigation technology", Stevenage, United Kingdom : Institution of Electrical Engineers, cop. 1997.

[10] Lamon, P., Krebs, A., Lauria, M., Shooter, S. and Siegwart, R., "Wheel torque control for a rough terrain rover", In Proceedings of the International Conference on Robotics and Automation, New Orleans (USA), 2004, in press.

[11] A. Mallet, S. Lacroix and L. Gallo, "Postion Estimation in Outdoor Environments using Pixel Tracking and Stereovision", In International Conference on Robotics and Automation, pages 3519-3524. San Francisco, CA (USA), 2000.

[12] I-K. Jung and S. Lacroix, "A robust Interest Point Matching Algorithm", In International Conference on Computer Vision. Vancouver (Canada), 2001.

[13] I-K. Jung and S. Lacroix, "Simultaneous Localization and Mapping with Stereovision", International Symposium on Robotics Research, Siena, Oct 2003 